University of Wollongong

Research Online

$1-1-2019$

\title{
Bereavement support in palliative care: a national survey of Australian
} services

Conrad Kobel

University of Wollongong, ckobel@uow.edu.au

Darcy Morris

University of Wollongong, darcy@uow.edu.au

Cristina J. Thompson

University of Wollongong, cthompso@uow.edu.au

Kate Williams

University of Wollongong, kathrynw@uow.edu.au

Follow this and additional works at: https://ro.uow.edu.au/ahsri

Research Online is the open access institutional repository for the University of Wollongong. For further information contact the UOW Library: research-pubs@uow.edu.au 


\title{
Bereavement support in palliative care: a national survey of Australian services
}

\author{
Abstract \\ Background: Bereavement support is an integral part of palliative care. However, audits of Australian \\ palliative care services have consistently identified bereavement care as one of the highest priorities for \\ improvement.
}

Objective: We assessed equity of access to bereavement support across Australian palliative care services by using survey data to compare services according to location (metropolitan vs. regional). We also evaluated changes in bereavement support over the last decade by comparing findings to results of a previous Australian study.

Design, Setting, and Participants: A national, cross-sectional online survey of Australian palliative care services conducted from August to September 2017.

Main Outcome Measures: Services' self-reported provision of bereavement support.

Results: One hundred and eighty services (84\%) responded. Of these, $91 \%$ provided bereavement support. Most offered support to all bereaved persons connected to the service. More than $80 \%$ of services provided a wide range of support types. Metropolitan services were more likely than regional services to offer specialist bereavement interventions. The staff most involved in coordinating and delivering bereavement support were social workers, nurses (particularly in regional areas), and bereavement coordinators/counselors (particularly in metropolitan areas). Resource limitations presented barriers to provision of bereavement support.

Conclusions: Across Australia, in principle, access to bereavement support through palliative care services remains largely equitable. Nevertheless, observed variations in the type of professional delivering care and the level of support indicate that a more consistent approach is required. An increase in the range of supports available compared with a decade ago signifies a more comprehensive approach to bereavement support by many Australian palliative care services.

\section{Publication Details}

C. Kobel, D. Morris, C. Thompson \& K. E. Williams, "Bereavement support in palliative care: a national survey of Australian services", Journal of Palliative Medicine 228 (2019) 933-938. 
Bereavement support in palliative care: a national survey of Australian services

Conrad Kobel, PhD, MFinMath ${ }^{1}$

Darcy Morris, MPH(Dist), GCertHlthRes, BA(Dist) ${ }^{1}$

Cristina Thompson, RN, RM, BA, MBA ${ }^{1}$

Kathryn E. Williams, PhD, BA(Hons) ${ }^{1}$

${ }^{1}$ Centre for Health Service Development, Australian Health Services Research Institute (AHSRI), University of Wollongong, Wollongong, New South Wales 2522, Australia

Corresponding author: Conrad Kobel (kobel@uow.edu.au) 
Bereavement support in palliative care: a national survey of Australian services 


\section{Abstract}

Background: Bereavement support is an integral part of palliative care. However, audits of Australian palliative care services have consistently identified bereavement care as one of the highest priorities for improvement.

Objective: We assessed equity of access to bereavement support across Australian palliative care services by using survey data to compare services according to location (metropolitan versus regional). We also evaluated changes in bereavement support over the last decade by comparing findings to results of a previous Australian study.

Design, setting and participants: A national, cross-sectional, online survey of Australian palliative care services conducted in August-September 2017.

Main outcome measures: Services’ self-reported provision of bereavement support.

Results: 180 services (84\%) responded. Of these, 91\% provided bereavement support. Most offered support to all bereaved persons connected to the service. More than $80 \%$ of services provided a wide range of support types. Metropolitan services were more likely than regional services to offer specialist bereavement interventions. The staff most involved in coordinating and delivering bereavement support were social workers, nurses (particularly in regional areas) and bereavement coordinators/counsellors (particularly in metropolitan areas). Resource limitations presented barriers to provision of bereavement support.

Conclusions: Across Australia, in principle access to bereavement support via palliative care services remains largely equitable. Nevertheless, observed variations in the type of professional delivering care and the level of support indicate that a more consistent approach is required. An increase in the range of supports available compared with a decade ago signifies a more comprehensive approach to bereavement support by many Australian palliative care services. 


\section{Introduction}

Around one in four deaths in Australia occurs in palliative care services. ${ }^{1}$

Bereavement support is seen as an integral part of palliative care ${ }^{2,3}$ in keeping with the principle of family-centred care. ${ }^{4}$ Carers who are bereaved in a palliative care setting appreciate and benefit from contact with the service after the death. ${ }^{5}$ Internationally, palliative care services have been recognised as providing "the most coherent approach" 6 while in Australia they are seen as a "driving force" for bereavement support. ${ }^{7}$ Palliative care services provide a leading pathway into bereavement support for families and friends of the deceased. ${ }^{8}$

Nevertheless, bereavement care has been consistently identified among the highest priorities for improvement in audits of Australian palliative care services. ${ }^{4,9}$

Internationally, the development of bereavement support services has not kept pace with the development of other aspects of palliative care. ${ }^{10}$ Services face challenges when providing family-centred care ${ }^{11}$ including resource constraints on the provision of care after the patient's death. ${ }^{12}$

A public health approach to bereavement support has been proposed in order to help services respond to these challenges and achieve better health outcomes. ${ }^{13}$ This approach involves targeting support according to identified need. Most people adapt to their loss over time, with the help of informal supports; for these individuals, interventions may be less effective. ${ }^{14}$ Instead, palliative care services should provide a universal, coordinated approach to bereavement support at various time points, to be taken up as required ${ }^{2}$ or, as Larson and Hoyt recommend, when offering grief counselling (and other support) to prospective clients, services should 'reach but not grab'. ${ }^{15}$ Information, screening and risk assessment is also recommended ${ }^{2}$ because for a small proportion of the population ${ }^{16}$ bereavement can cause intense and persistent symptoms of distress leading to severe mental and physical health problems. ${ }^{17}$ People at risk of, or suffering from, prolonged grief disorder ${ }^{18}$ require identification and referral to specialist, tailored bereavement support interventions. ${ }^{2,19}$

In 2007, a survey of 236 Australian palliative care services found that 95\% provided some form of bereavement support, $96 \%$ of metropolitan and $94 \%$ of regional services. ${ }^{20}$ This study replicates and extends this work by examining the bereavement support provided by Australian palliative care services a decade later. It examines prevalence, staffing and types of support, explores factors (including geographic location) that may impact service provision, and compares findings with previous studies $^{12,20}$ to identify changes in bereavement support. The study was one component of a larger research project into services for and needs of people experiencing bereavement and prolonged grief.

\section{Methods}

\section{Participants and sample}

A list of palliative care services was created from all members (data submitting and other services, $\mathrm{n}=198$ ) of the Palliative Care Outcomes Collaboration (PCOC) (www.pcoc.org.au) and all "specialist palliative care providers” (219 of 362 entries) 
from the Palliative Care Australia (PCA) Directory of Services ${ }^{21}$ (individual persons, support agencies and corporate headquarters were removed). Most services were telephoned to verify appropriate contact details and encourage participation. In total, 215 invitations were emailed with personalised web links in early August 2017. Two reminders were sent to non-respondents at fortnightly intervals. PCOC Facilitators encouraged services to participate in the survey via email. The survey closed in midSeptember 2017. Completion of the survey was regarded as implied consent.

\section{Survey}

The survey was based on a previous survey ${ }^{20}$ with additional questions derived from Bereavement Support Standards for Specialist Palliative Care Services. ${ }^{2}$ Twelve individuals with a range of expertise pilot-tested the survey, resulting in minor revisions. The final survey instrument included 32 questions. However, due to adaptive questioning, each respondent was asked between 12 to 27 questions. The survey was implemented using the Qualtrics research platform and respondents were able to review and change their answers prior to submission. Only the project team had access to the downloaded survey data. All survey responses (partially and fully completed) were included in the analysis if the question "Does your service provide bereavement follow-up/support?” had been answered.

\section{Data analysis}

Responses were analysed using descriptive statistics (including absolute and relative frequencies). Chi-squared tests were used to assess differences in the frequency of responses between services based on location (metropolitan versus regional). While the survey included the options "metropolitan", "regional" and "remote", there were limited remote services ( $n=7)$ and consequently low numbers of responses, thus results for regional and remote areas of service were combined and are referred to as regional in this article. P-values smaller than 0.05 were considered statistically significant. Content analysis of open-ended questions was conducted to identify common themes. Data analysis was performed in Excel and SAS 9.4.

\section{Ethics approval}

The project was approved by the University of Wollongong and Illawarra Shoalhaven Local Health District Social Sciences Human Research Ethics Committee (2017/316).

\section{Results}

Of the 215 invited palliative care services, 180 provided valid responses. The response rate (84\%) was high across all Australian states and territories, ranging from $79 \%$ in South Australia to 100\% in Tasmania, the Northern Territory and the Australian Capital Territory.

Table 1 provides an overview of the study sample. Almost three quarters of responses were from services in New South Wales, Victoria and Queensland and more than half of services (58\%) served regional (including remote) areas. Sixty percent of services were either integrated (inpatient and community) services or inpatient services (including hospital-based consultation/liaison services). Around one third of services were community services (including community-based consultation/liaison services). The remaining “other” services included minor variations of these response 
categories. Service size was classified by the number of patient deaths during a sixmonth period, ranging from very small services (fewer than 20) to large services (greater than 100). Services were also categorised according to their PCA service level $^{22}$ which provides a framework ranging from primary-care based services through three levels of specialist services, with Level 3 representing the highest level of capability and resources.

In total, 163 (91\%) palliative care services provided bereavement support, with similar proportions within metropolitan (92\%) and regional (89\%) services. Seventeen services did not provide bereavement support but would assist bereaved individuals as needed. Seven of those services indicated that they intended to develop a bereavement program.

Services providing bereavement support were subsequently asked about the types of care provided at six time points on the grief trajectory (158 services responded), reported in Table 2. The most frequent type of bereavement support was brief followup contact with bereaved families / significant others (e.g. phone call, letter, email). In metropolitan services this was followed by risk assessment for prolonged grief, specialist bereavement intervention (either in-house or by referral), provision of written bereavement information about psychological and emotional support, remembrance activities (e.g. anniversary card, invitation to memorial service) and provision of information about legal, financial and other practical issues. In regional services this was followed by provision of information about legal, financial and other practical issues, risk assessment for prolonged grief, specialist bereavement intervention (either in-house or by referral) and access to support sessions/visits (individual or group). All these supports were provided by more than $80 \%$ of services.

The proportion of metropolitan services providing specialist bereavement intervention (either in-house or by referral) (94\%) was significantly higher $(\mathrm{p}=0.045)$ than regional services (84\%). Most service delivery occurred "as soon as practicable following death" with 90-92\% of all services undertaking at least one type of support at that time point. At the earliest time point investigated, "between admission and the patient's death”, the proportion of regional services (90\%) providing bereavement support was significantly higher $(p=0.017)$ than metropolitan services $(76 \%)$.

In $80 \%$ of services, bereavement support was coordinated by either a social worker, nurse or bereavement coordinator/counsellor (Table 3). Bereavement coordinators/counsellors were more likely to take the coordination role in metropolitan services than regional services $(p<0.01)$. Nurses, on the other hand, were more likely to take the coordination role in regional services than metropolitan services ( $\mathrm{p}<0.01$ ). Delivery of bereavement support involved a broader range of staff, more so in metropolitan services than regional services. Significant differences between metropolitan and regional services were observed for nurses, bereavement coordinators/counsellors and pastoral care workers involved in bereavement support delivery ( $\mathrm{p}<0.01$ ); with higher nurse involvement in regional services and higher bereavement coordinator/counsellor and pastoral care worker involvement in metropolitan services. 
Overall, 91\% of services performed risk assessment for prolonged grief, most commonly by multidisciplinary team opinion (63\%). In metropolitan services this was followed by single staff member opinions (49\%) and use of formal bereavement risk assessment tools (43\%) whereas in regional services the order was reversed (51\% and $36 \%$ respectively).

When asked to whom bereavement support was provided, most services (82\%) reported that support was offered to bereaved persons of all palliative care patients. Additionally, bereavement support was offered to persons identified as high risk (by $39 \%$ of metropolitan and $43 \%$ of regional services) and self-referred persons (by $42 \%$ and 34\% respectively). Fifteen percent of services also offered bereavement support to other groups. To meet the needs of the bereaved, around $73 \%$ of services employed Aboriginal health staff and $45 \%$ had bilingual health staff available; both with slightly higher rates in metropolitan services than in regional services.

The most commonly cited barriers to providing bereavement support related to resource limitations: insufficient staff time; lack of personnel; and funding pressures, including concern about undertaking work that was not counted in existing activitybased funding models. Human resource issues were particularly problematic for regional services, as was lack of appropriate services to which bereaved persons could be referred and difficulties associated with covering large geographic areas.

\section{Discussion}

With a high response rate of $84 \%$, this survey provides a nationally representative sample of Australian palliative care services. Several factors may have contributed to the high response rate including online administration and prior phone contact with services.

Ninety-one percent of services provided bereavement support and all others indicated that they assisted bereaved persons if necessary. Hence, in practice, all services provide some form of bereavement support. This is higher than the proportion reported in a large study of European countries which found $82 \%(n=302)$ of services provide bereavement support ${ }^{23}$ but consistent with an earlier Australian study. ${ }^{20}$

The current study demonstrates that bereavement support is accessible through Australian palliative care services irrespective of the geographic location. However, access to palliative care services more broadly is an issue in Australia. Despite being a core primary health service to which all Australians should have access, ${ }^{24}$ "palliative care services are currently not able to meet the needs of everyone in our community, particularly groups with diverse needs [including] regional and remote residents" ${ }^{25}$ A recent literature review found "palliative care patients living in regional and remote areas of Australia experience poor access and [...] cannot access services within their local community because such services do not exist”. ${ }^{26}$ Thus, limited access to palliative care services in regional and remote Australia inevitably limits access to bereavement support provided by these services. Nonetheless, it is important to recognise that palliative care services are not the sole providers of bereavement support. Future studies focusing specifically on assessing the unique challenges for remote palliative care services in the provision of bereavement support are warranted. 
While the proportion of services providing bereavement support has remained high over the past decade, some change is evident in the types of support available. For example, $94 \%$ of services now provide psychosocial information and 79\% provide practical information, compared with just $5 \%$ of services providing an information package in the previous study. ${ }^{20}$ There has been an increase in the prevalence of remembrance activities, which are now provided by $82 \%$ of services (compared with $66 \%$ and 53\% providing memorials or anniversary cards, respectively). ${ }^{20}$ Further, a wider range of supports is now available, with most types of supports provided by at least $80 \%$ of services. This appears to signify a shift towards a more comprehensive approach to bereavement support by palliative care services.

Social workers have a prominent role in coordinating and delivering bereavement support, as do nurses (particularly in regional services). The role of bereavement coordinators/counsellors has expanded in recent years (primarily in metropolitan services), with services reporting higher involvement in coordination (25\%) and delivery (38\%), up from $19 \%$ and $22 \%$ a decade ago. ${ }^{20}$ While volunteers continue to play an important role in delivering bereavement support, pastoral care workers are now involved in $26 \%$ of services compared with $31 \%$ previously. ${ }^{20}$ More services are reporting the involvement of psychologists and medical doctors in delivering bereavement support, compared with the earlier survey. Similar patterns of staff involvement in delivering bereavement support are evident over the past decade. Mather and colleagues found social workers, nurses and pastoral care workers were most involved, with nurses by far most frequently involved in regional services. ${ }^{20}$ This highlights the fact that the professionals coordinating and delivering bereavement support differ significantly while in principle access to (any) support in metropolitan and regional services is similar. Currently, bereavement coordinators/counsellors and social workers are most involved in metropolitan services, while nurses and social workers are most involved in regional services. This finding, especially the prominence of nurses and social workers in bereavement support, has implications for education and support. Improved access to formal education and support for staff in their role in bereavement support is needed and has been identified previously. ${ }^{4,12,20}$

Few significant differences between metropolitan and regional services were observed. Overall, the bereavement supports provided were similar with the exception of specialist intervention (either in-house or by referral) which was more frequently provided by metropolitan services. Additional analyses confirmed this picture. Although larger services had a wider range of staff available to coordinate and deliver bereavement support, this was not reflected in significant differences in the support actually provided. Further analyses of the services' characteristics revealed significant levels of association between area of service, service size, service setting and PCA level. Hence, some observed differences between metropolitan and regional services may be due to differences other than only the area of service.

Ten years ago, Abbott et al. identified the main obstacles in delivering bereavement and palliative care services as "lack of sufficient staff time, followed by funding pressures, lack of personnel, and lack of organisational support of bereavement services". ${ }^{12}$ More recently, Hudson et al. characterised bereavement support by 
palliative care as "typically insufficiently resourced, under-researched, and not systematically applied". ${ }^{17}$ These obstacles and characterisations were largely consistent with those reported by metropolitan and regional services alike in the present study, suggesting long-standing challenges persist. An additional concern reported by several services in this study related to difficulties in providing ongoing support as bereaved persons are not clients of a service.

The proportion of palliative care services offering bereavement support to the bereaved of all palliative patients is now $82 \%$; almost unchanged in the past decade. Earlier studies reported between $83 \%{ }^{20}$ and $94 \%{ }^{12}$ of services. While universal access to bereavement support is recommended in standards for Victorian specialist palliative care services, ${ }^{2}$ it is different from universal provision; the latter risks spreading limited resources too thinly to be effective. ${ }^{6,27}$

The alternative (public health) approach involves allocating resources according to identified need, with specialist bereavement interventions reserved for those at risk of, or suffering from, prolonged grief disorder. ${ }^{13}$ Palliative care services should implement multiple strategies to identify these individuals, including a variety of assessment types (e.g. conversational exploration of risk factors; validated tools) and follow-up contacts at various time points in the grief trajectory. ${ }^{2}$ There are indications that the palliative care sector in Australia is moving towards this model. Currently, $91 \%$ of services undertake some form of risk assessment (most frequently multidisciplinary team opinion), an increase from $69 \%$ reported by Mather et al. ${ }^{20}$ This encouraging finding aligns with elements of the National Palliative Care Standards; specifically an interdisciplinary team approach and an emphasis on clinical assessment of carers and family., ${ }^{42}$ However, barriers relating to bereavement risk assessment in palliative care have been identified ${ }^{28}$ as have issues with risk assessment measures. ${ }^{27,29}$ Development of new models of assessment are promising. ${ }^{30,31}$ Primary health care providers also have important roles to play in supporting a public health approach to bereavement support. ${ }^{27}$

This study assessed equity of access to bereavement support across Australian palliative care services by examining the support provided and identifying differences between metropolitan and regional services. It compared findings with previous research. The survey relied on self-reporting by services. Although the response rate was high, findings may be biased by the possibility that services which did not provide bereavement support may have been less likely to respond. The study was limited by the practical need for survey brevity, restricting the range of issues that could be explored further. For example, the survey did not collect information on whether risk assessment led to follow-up by services. Actions resulting from risk assessment is an important area for future research. Additionally, calls for further research to ensure bereavement support is supported by a rigorous evidence-base ${ }^{7,32}$ remain pertinent, including examining outcomes associated with support provided by palliative care services in routine practice.

The vast majority of Australian palliative care services continue to acknowledge bereavement support as a core responsibility. Palliative care policy documents (e.g. National Palliative Care Strategy ${ }^{33}$ and National Palliative Care Standards ${ }^{22,34}$ ) have 
likely contributed to this recognition and it is therefore crucial to ensure the continued prominence of bereavement support in such documents.

Overall, palliative care services are providing a more comprehensive range of supports than previously, but the availability of support varies considerably between services. While bereavement support is offered by almost all services irrespective of geographic location, the professional providing the support differs significantly between metropolitan and regional services. As such, development of national bereavement support standards promoting more consistent and better targeted support may enhance bereavement care provision by palliative care services, recognising significant work is already underway in this area (for example, by Hudson and colleagues $^{17,35}$ ).

\section{Acknowledgements}

The project was funded by the Australian Government Department of Health. The views expressed in this article do not necessarily reflect the views of the Australian Government. We wish to particularly thank Dr Mark Mather and colleagues for providing information about the survey they undertook in 2007 and allowing us to replicate questions from this survey. Appreciation is also extended to our colleagues in the Palliative Care Outcomes Collaboration for their guidance and advice.

\section{Author Disclosure Statement}

No competing financial interests exist. 


\section{References}

1. Eagar K, Clapham S, Allingham S: No, most people aren’t in severe pain when they die. The Conversation, 11 Dec 2017.

https://theconversation.com/no-most-people-arent-in-severe-pain-when-theydie-86835 (accessed Jan 2019).

2. Hall C, Hudson P, Boughey A: Bereavement support standards for specialist palliative care services. Melbourne: Department of Health, State Government of Victoria, 2012.

3. Daubman BR: My Condolences: Making Bereavement Contact Part of Our Standard Operating Procedures. J Palliat Med 2018;21:1058-1059.

4. Palliative Care Australia: National Standards Assessment Program: Quality Report 2010 - 2015. Canberra: Palliative Care Australia, 2016.

5. Makarem M, Mohammed S, Swami N et al.: Experiences and Expectations of Bereavement Contact among Caregivers of Patients with Advanced Cancer. $J$ Palliat Med 2018;21:1137-1144.

6. Aoun SM, Rumbold B, Howting D, et al.: Bereavement support for family caregivers: The gap between guidelines and practice in palliative care. PLoS One 2017;12:e0184750.

7. Waller A, Turon H, Mansfield E, et al.: Assisting the bereaved: A systematic review of the evidence for grief counselling. Palliat Med 2016;30:132-148.

8. Thompson C, Williams K, Masso M, et al.: Research into services and needs for people experiencing complicated grief: Final report. Wollongong: Centre for Health Service Development, Australian Health Services Research Institute, University of Wollongong, 2017.

9. Palliative Care Australia: National Standards Assessment Program: National Quality Report. Canberra: Palliative Care Australia, 2011.

10. Morris SE, Block SD: Adding Value to Palliative Care Services: The Development of an Institutional Bereavement Program. J Palliat Med 2015;18:915-22.

11. Kissane DW: The challenge of family-centered care in palliative medicine. Ann Palliat Med 2016;5:319-321. 
12. Abbott J, O’Connor M, Payne S: An Australian survey of palliative care and hospice bereavement services. Aust J Cancer Nurs 2008;9:12-17.

13. Aoun SM, Breen LJ, O'Connor M, et al.: A public health approach to bereavement support services in palliative care. Aust N Z J Public Health 2012;36:14-16.

14. Currier JM, Neimeyer RA and Berman JS: The effectiveness of psychotherapeutic interventions for bereaved persons: a comprehensive quantitative review. Psychol Bull 2008;134:648-661.

15. Larson DG, Hoyt WT: Grief counselling efficacy. Bereavement Care 2009;28:14-19.

16. Lundorff M, Holmgren $H$, Zachariae R, et al.: Prevalence of prolonged grief disorder in adult bereavement: A systematic review and meta-analysis. $J$ Affect Disord 2017;212:138-149.

17. Hudson P, Hall C, Boughey A, Roulston A: Bereavement support standards and bereavement care pathway for quality palliative care. Palliat Support Care 2017;doi:10.1017/S1478951517000451.

18. Maciejewski PK, Prigerson HG: Prolonged, but not complicated, grief is a mental disorder. Br J Psychiatry 2017;211:189-191.

19. Hall C: Bereavement theory: recent developments in our understanding of grief and bereavement. Bereave Care 2014;33:7-12.

20. Mather MA, Good PD, Cavenagh JD, Ravenscroft PJ: Survey of bereavement support provided by Australian palliative care services. Med J Aust 2008;188:228-230.

21. Palliative Care Australia: Directory of Services. http://palliativecare.org.au/directory-of-services/ (accessed Jul 2017).

22. Palliative Care Australia: National Palliative Care Standards, 5th Edition. Canberra: Palliative Care Australia, 2018.

23. Guldin MB, Murphy I, Keegan, O, et al.: Bereavement care provision: A survey by the EAPC Bereavement Care Taskforce. Eur J Palliat Care 2015;22:185-189. 
24. Thomas SL, Wakerman, Humphreys JS: Ensuring equity of access to primary health care in rural and remote Australia - what core services should be locally available? Int J Equity Health 2015;14:111.

25. Palliative Care Australia: Submission to the Senate Select Committee on Health. Canberra: Palliative Care Australia, 2014.

26. Jansson M, Dixon K, Hatcher D: The palliative care experiences of adults living in regional and remote areas of Australia: A literature review. Contemp Nurse 2017;53:94-104.

27. Breen LJ, Aoun SM, O'Connor M, Rumbold B: Bridging the Gaps in Palliative Care Bereavement Support: An International Perspective. Death Stud 2014;38:54-61.

28. Sealey M, O’Connor M, Aoun SM, Breen LJ: Exploring barriers to assessment of bereavement risk in palliative care: perspectives of key stakeholders. BMC Palliat Care 2015;14:49.

29. Sealey M, Breen LJ, O'Connor M, Aoun SM: A scoping review of bereavement risk assessment measures: Implications for palliative care. Palliat Med 2015;29:577-589.

30. Blackburn P, Dwyer K: A Bereavement Common Assessment Framework in Palliative Care: Informing Practice, Transforming Care. Am J Hosp Palliat Care 2017;34:677-684.

31. Roberts K, Holland J, Prigerson HG, et al.: The Development of the Bereavement Risk Inventory and Screening Questionnaire (BRISQ): Item Generation and Expert Panel Feedback. Palliat Support Care 2017;15:57-66.

32. Forte AL, Hill M, Pazder R, Feudtner C: Bereavement care interventions: a systematic review. BMC Palliat Care 2004;3:3.

33. Commonwealth of Australia: National Palliative Care Strategy 2010. Canberra: Commonwealth of Australia, 2010.

34. Palliative Care Australia: Standards for Providing Quality Palliative Care for all Australians. Canberra: Palliative Care Australia, 2005.

35. Hudson P, Remedios C, Zordan R, et al.: Guidelines for the psychosocial and bereavement support of family caregivers of palliative care patients. $J$ Palliat Med 2012;15:696-702. 
Table 1 Characteristics of palliative care services

\begin{tabular}{|c|c|c|}
\hline Characteristic & $\mathbf{n}$ & $(\%)$ \\
\hline \multicolumn{3}{|l|}{ State / Territory $(\mathbf{n}=180)$} \\
\hline New South Wales & 56 & $(31 \%)$ \\
\hline Victoria & 47 & $(26 \%)$ \\
\hline Queensland & 30 & $(17 \%)$ \\
\hline South Australia & 15 & $(8 \%)$ \\
\hline Western Australia & 22 & $(12 \%)$ \\
\hline Tasmania & 6 & $(3 \%)$ \\
\hline Northern Territory & 2 & $(1 \%)$ \\
\hline Australian Capital Territory & 2 & $(1 \%)$ \\
\hline \multicolumn{3}{|l|}{ Area of service $(n=180)$} \\
\hline Metropolitan & 76 & $(42 \%)$ \\
\hline Regional & 104 & $(58 \%)$ \\
\hline \multicolumn{3}{|l|}{ Service setting $(n=180)$} \\
\hline Integrated (inpatient and community) service & 62 & (34\%) \\
\hline Community service & 58 & $(32 \%)$ \\
\hline Inpatient service & 46 & $(26 \%)$ \\
\hline Other & 14 & $(8 \%)$ \\
\hline \multicolumn{3}{|l|}{ Service size $(n=180)$} \\
\hline Very small (less than 20 patient deaths in 6 months) & 18 & $(10 \%)$ \\
\hline Small (20-49 patient deaths in 6 months) & 45 & $(25 \%)$ \\
\hline Medium (50-99 patient deaths in 6 months) & 49 & $(27 \%)$ \\
\hline Large (100 or more patient deaths in 6 months) & 68 & $(38 \%)$ \\
\hline \multicolumn{3}{|l|}{ Palliative Care Australia Specialist Level $(\mathrm{n}=178)$} \\
\hline Primary Care Service & 9 & $(5 \%)$ \\
\hline Specialist Palliative Care Level 1 & 45 & $(25 \%)$ \\
\hline Specialist Palliative Care Level 2 & 45 & $(25 \%)$ \\
\hline Specialist Palliative Care Level 3 & 54 & $(30 \%)$ \\
\hline Unknown & 25 & $(14 \%)$ \\
\hline
\end{tabular}


Table 2 Types and timing of bereavement support

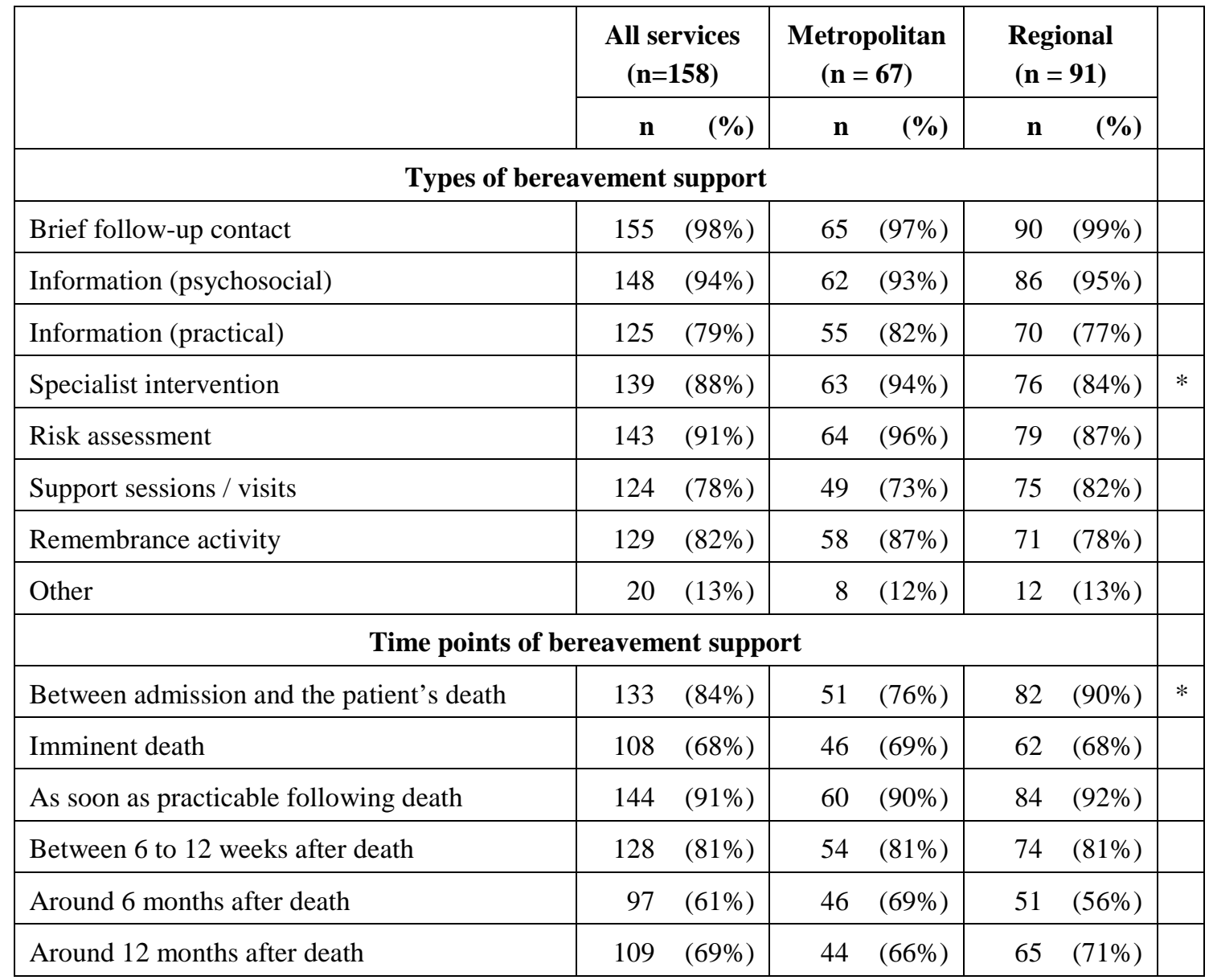

* indicates statistically significant result 
Table 3 Staff involved in coordination and delivery of bereavement support

\begin{tabular}{|c|c|c|c|c|c|c|c|c|c|c|c|c|c|c|}
\hline \multirow{3}{*}{ Staff description } & \multicolumn{7}{|c|}{ Coordination } & \multicolumn{7}{|c|}{ Delivery } \\
\hline & \multicolumn{2}{|c|}{$\begin{array}{l}\text { All services } \\
(\mathrm{n}=154)\end{array}$} & \multicolumn{2}{|c|}{$\begin{array}{c}\text { Metropolitan } \\
(n=64)\end{array}$} & \multicolumn{2}{|c|}{$\begin{array}{c}\text { Regional } \\
(\mathbf{n}=90)\end{array}$} & & \multicolumn{2}{|c|}{$\begin{array}{c}\text { All services } \\
(n=154)\end{array}$} & \multicolumn{2}{|c|}{$\begin{array}{c}\text { Metropolitan } \\
(\mathrm{n}=64)\end{array}$} & \multicolumn{2}{|c|}{$\begin{array}{c}\text { Regional } \\
(\mathbf{n}=90)\end{array}$} & \\
\hline & $\mathbf{n}$ & $(\%)$ & $\mathbf{n}$ & $(\%)$ & $\mathrm{n}$ & (\%) & & $\mathbf{n}$ & (\%) & $\mathbf{n}$ & (\%) & $\mathbf{n}$ & (\%) & \\
\hline Social worker & 44 & $(29 \%)$ & 18 & $(28 \%)$ & 26 & $(29 \%)$ & & 98 & $(64 \%)$ & 43 & $(67 \%)$ & 55 & $(61 \%)$ & \\
\hline Nurse & 40 & $(26 \%)$ & 8 & $(13 \%)$ & 32 & $(36 \%)$ & $*$ & 96 & $(62 \%)$ & 28 & $(44 \%)$ & 68 & $(76 \%)$ & * \\
\hline Bereavement coordinator / counsellor & 39 & $(25 \%)$ & 24 & $(38 \%)$ & 15 & $(17 \%)$ & * & 58 & $(38 \%)$ & 34 & $(53 \%)$ & 24 & $(27 \%)$ & * \\
\hline Pastoral care worker & 11 & $(7 \%)$ & 6 & (9\%) & 5 & $(6 \%)$ & & 40 & $(26 \%)$ & 25 & $(39 \%)$ & 15 & $(17 \%)$ & $*$ \\
\hline Volunteer & 3 & $(2 \%)$ & 0 & (0\%) & 3 & (3\%) & & 44 & (29\%) & 15 & (23\%) & 29 & $(32 \%)$ & \\
\hline Psychologist & 1 & $(1 \%)$ & 0 & $(0 \%)$ & 1 & $(1 \%)$ & & 20 & $(13 \%)$ & 11 & $(17 \%)$ & 9 & $(10 \%)$ & \\
\hline Doctor & 0 & $(0 \%)$ & 0 & $(0 \%)$ & 0 & $(0 \%)$ & & 22 & $(14 \%)$ & 12 & $(19 \%)$ & 10 & $(11 \%)$ & \\
\hline Other & 16 & $(10 \%)$ & 8 & (13\%) & 8 & (9\%) & & 18 & (12\%) & 5 & $(8 \%)$ & 13 & $(14 \%)$ & \\
\hline
\end{tabular}

* indicates statistically significant result 